\title{
The evolutionary trajectory of the mating-type (mat) genes in Neurospora relates to reproductive behavior of taxa Lotta Wik ${ }^{1}$, Magnus Karlsson ${ }^{2}$ and Hanna Johannesson*3
}

Address: ${ }^{1}$ Swedish University of Agricultural Sciences, Department of Biomedical Sciences and Veterinary Public Health, Husargatan 3, SE-75123 Uppsala, Sweden, 2 Swedish University of Agricultural Sciences, Department of Forest Mycology and Pathology, Ulls väg 26, SE-750 07 Uppsala, Sweden and ${ }^{3}$ Uppsala University, Department of Evolutionary Biology, Norbyvägen 18D, SE-752 36 Uppsala, Sweden

Email: Lotta Wik - lotta.wik@bvf.slu.se; Magnus Karlsson - Magnus.Karlsson@mykopat.slu.se;

Hanna Johannesson* - hanna.johannesson@ebc.uu.se

* Corresponding author

Published: II April 2008

BMC Evolutionary Biology 2008, 8:109 doi:10.1186/147/-2148-8-109

This article is available from: http://www.biomedcentral.com/147I-2/48/8/109

(c) 2008 Wik et al; licensee BioMed Central Ltd.

This is an Open Access article distributed under the terms of the Creative Commons Attribution License (http://creativecommons.org/licenses/by/2.0), which permits unrestricted use, distribution, and reproduction in any medium, provided the original work is properly cited.
Received: 12 October 2007

Accepted: II April 2008

\begin{abstract}
Background: Comparative sequencing studies among a wide range of taxonomic groups, including fungi, have led to the discovery that reproductive genes evolve more rapidly than other genes. However, for fungal reproductive genes the question has remained whether the rapid evolution is a result of stochastic or deterministic processes. The mating-type (mat) genes constitute the master regulators of sexual reproduction in filamentous ascomycetes and here we present a study of the molecular evolution of the four mat-genes (mat a-I, mat A-I, mat A-2 and mat A-3) of 20 Neurospora taxa.
\end{abstract}

Results: We estimated nonsynonymous and synonymous substitution rates of genes to infer their evolutionary rate, and confirmed that the mat-genes evolve rapidly. Furthermore, the evolutionary trajectories are related to the reproductive modes of the taxa; likelihood methods revealed that positive selection acting on specific codons drives the diversity in heterothallic taxa, while among homothallic taxa the rapid evolution is due to a lack of selective constraint. The latter finding is supported by presence of stop codons and frame shift mutations disrupting the open reading frames of mat a-I, mat A-2 and mat A-3 in homothallic taxa. Lower selective constraints of matgenes was found among homothallic than heterothallic taxa, and comparisons with nonreproductive genes argue that this disparity is not a nonspecific, genome-wide phenomenon.

Conclusion: Our data show that the mat-genes evolve rapidly in Neurospora. The rapid divergence is due to either adaptive evolution or lack of selective constraints, depending on the reproductive mode of the taxa. This is the first instance of positive selection acting on reproductive genes in the fungal kingdom, and illustrates how the evolutionary trajectory of reproductive genes can change after a switch in reproductive behaviour of an organism.

\section{Background}

Rapidly evolving genes are those with a higher than average percentage of amino-acid substitutions between species. Rapid evolution may be due to adaptive evolution, which occurs when natural selection promotes aminoacid divergence. Alternatively, rapid evolution may be due to a lack of functional constraint; for example, a pseudog- 
ene rapidly accumulates mutations because of an absence of purifying selection.

Comparative sequencing studies among a wide range of taxonomic groups have led to the discovery that reproductive genes evolve more rapidly than other genes. In animals, essentially all steps in fertilization where the genes have been identified there is evidence of rapid divergence, and numerous reports of positively selected animal reproductive genes exist [1]. In contrast, in plants and fungi the selective forces driving reproductive gene diversity, and the functional consequences of reproductive gene evolution, are just beginning to be understood. In filamentous ascomycetes, comparatively low levels of between species nucleotide identity have been observed for mating-type genes, which constitute the master-regulators of sexual reproduction in this group of fungi [2]. Consequently these genes have been suggested to evolve at a faster rate than genes coding for e.g. metabolic enzymes [2-4], although the question has remained whether the rapid evolution is a result of stochastic or deterministic processes.

Many fungi have both asexual and sexual stages as a part of their life cycle. Their sexual mating systems are classified as either heterothallic or homothallic. In heterothallic fungi, strains must be of opposite mating type for sexual reproduction and morphogenesis, while homothallic species, generally thought to evolve from heterothallic ancestors [[5] and references therein], reproduce sexually without a mating partner. In all heterothallic filamentous ascomycetes examined to date, the mat locus is the sole determinant of mating type, and sexual development in heterothallic taxa is regulated by alternative mat A or mat $a$ genes at this locus. Alternate sequences at mat, denoted idiomorphs [6], lack significant sequence similarity and encode different transcriptional regulators [7-10]. Genetic analyses of the model species Neurospora crassa have identified one open reading frame (ORF) in the mat a idiomorph (mat a-1) as the major mating regulator in mat $a$ strains [8]. The mat $A$ idiomorph contains three genes, one of which is the main regulator of sexual development in mat A strains (mat A-1) [7,11]. While MAT A-1 and MAT a- 1 constitute the critical factors for both mating and sexual development in N. crassa, MAT A-2 and MAT A-3 are suggested to increase the efficiency of the process [12].

In contrast to many yeast species [13], filamentous ascomycetes of either mating type are unable to switch to the opposite mating type. Nevertheless, it has been suggested that conversion from heterothallic to homothallic mode in filamentous ascomycetes resides within the mat locus, and that homothallic species carry both idiomorphs in a single thallus [14]. In so called pseudohomothallic species, represented in this study by Neurospora tetrasperma, two haploid nuclei of opposite mating-type (mat $a$ and mat A) are maintained in each heterokaryotic ascospore progeny and vegetative cell, while in true homothallic species mat-genes from both idiomorphs are found within a single haploid genome. The structure of the mating type genes in homothallic Neurospora has not been determined in detail; however, hybridizations using cloned portions of the idiomorphs of $N$. crassa to the genome of related homothallic taxa have revealed three classes of homothallic taxa (Figure 1). The haploid genomes of one group contain both mat $a$ and mat $A$ sequences [15]. Another distinct group consists of taxa containing the entire mat $A$ sequence, while the mat $a-1$ gene is absent $[16,17]$. Finally, the hybridization patterns of the homothallic $N$. terricola provide evidence for the presence of mat a-1, mat A-1 and mat A-2 sequences, but it appears to lack parts of the A-idiomorphic region corresponding to the mat A-3 region [15].

Here we present the first, to our knowledge, extensive study of molecular evolution of fungal mating-type genes in relation to reproductive strategy. We sequenced the four mat-genes of Neurospora and Gelasinospora taxa, which we for simplicity refer to as Neurospora in this study. In addition, for comparative purposes we sequenced parts of three genes with function assumed to be independent of reproductive mode of fungi; encoding actin (act), translational elongation factor EF-1 alpha (tef-1) and glyceraldehyde 3-phosphate dehydrogenase (ccg-7). We confirm that the mat-genes evolve rapidly in this group of fungi and show that the evolutionary trajectory of each of the mat-genes is specifically related to the reproductive modes of the taxa; positive selection drives mat-gene diversity in heterothallic taxa, while the rapid evolution is due to a lack of selective constraint among homothallic taxa.

\section{Results}

\section{Sequence analysis of mat a-I}

Amplification products and sequences of mat $a-1$, originally described by Staben and Yanofsky [8], were successfully obtained from all investigated heterothallic strains of mating type a (mat a strains), and from all homothallic strains except $N$. africana, $N$. galapagosensis, $N$. dodgei and $N$. lineolata, thus confirming a previous report [17], suggesting that these four homothallic taxa lack the mat $a-1$ ORF. Taken together, mat $a-1$ was sequenced from twelve heterothallic and five homothallic strains, and the alignments of nucleotide and amino acid sequences of the gene are shown in Supplemental Figure S1 (Additional file 1). Several differences were found when comparing genomic and cDNA sequences of strain FGSC \#4200 with the originally published N. crassa sequence of the same strain [8]. The most striking difference was an additional cytosine found downstream from the conserved, DNA binding domain of the high mobility group (HMG), at nucleotide 


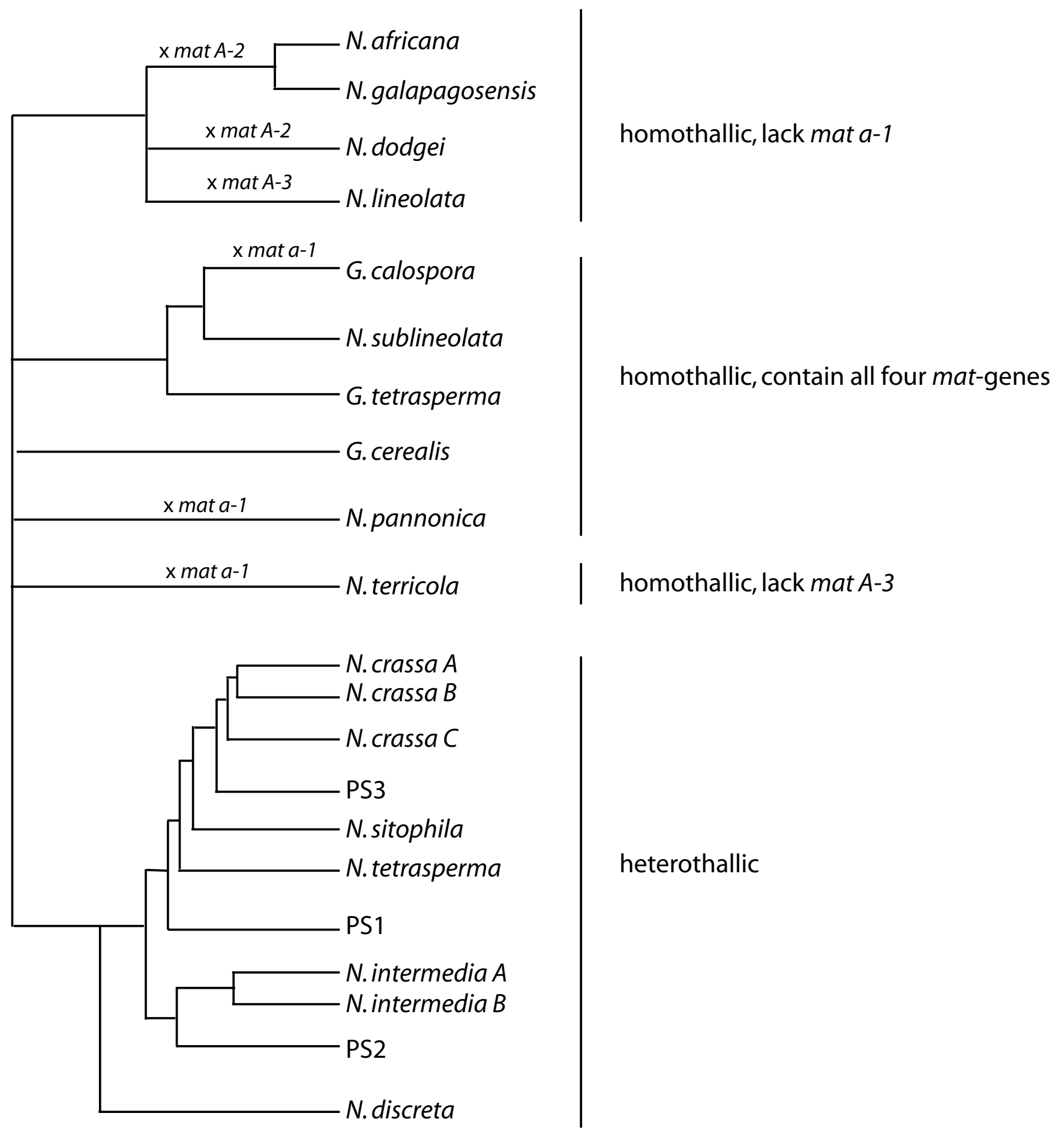

Figure I

Evolutionary relationship of the included taxa. Topology is constructed from previously reported topologies based on rDNA loci (28S, ITS), nuclear gene loci (Bml, ccg-7, mat a-I, mat A-I), and four anonymous nuclear loci [36-38, 46]. A cross indicates lineages where any of the ORFs is disrupted. A, B and C indicates intraspecific subgroups and PSI through 3 phylogenetic species [46]. 
position 1074, resulting in a shift in the ORF at amino acid position 359. Reading the resulting sequence until the first stop-codon results in a 438 amino acid MAT a-1 polypeptide in N. crassa instead of 381, as was originally suggested by Staben and Yanofsky [8]. Consequently, the $\mathrm{C}$-terminal part of the protein is different from that of the previously reported MAT a-1 protein. This feature of FGSC \#4200 was consistently found in all strains analyzed, supporting our revised description of mat $a-1$.

The size of the ORF of mat a-1 was found to be identical in all heterothallic taxa included in this study, and only a few amino acid substitutions were found. However, this was not the case among the six homothallic taxa, especially in the C-terminal part in which insertions and deletions of amino acids were found. In three of the homothallic taxa, N. pannonica, $N$. terricola and $G$. calospora, sequencing of both genomic and CDNA revealed disrupted mat a-1 ORFs (Figure 1). First, N. pannonica was found to lack a 125 bp region in the third exon (nucleotide position 826 to 965, Supplemental Figure S1 (Additional file 1)) located between two stretches of cytosine nucleotides conserved in the other taxa. This deletion in $N$. pannonica results in a frame shift introducing a premature stop codon 25 amino acids downstream of the deletion. In N. terricola, a stop codon was found at position 308 and in G. calospora an inserted adenosine that results in a frame shift at amino acid position 438 was found, although the HMG-box remained intact. Taken together, these data show that only two of the homothallic taxa included in this study possess a mat $a-1$ gene that is not modified.

\section{Sequence analyses of mat A-I}

Amplification products and sequences of mat A-1 were successfully obtained from the 12 heterothallic mat $A$ and the nine homothallic strains investigated here. The alignments of nucleotide and amino acid sequences of mat A-1 are shown in Supplemental Figure S1 (Additional file 1). We confirmed the 293 amino-acid mat A-1 sequence of reference strain FGSC\# 2489 of $N$. crassa, originally published by Glass et al. 1990 [7], including a DNA-binding $\alpha 1$ domain showing similarity to the $\alpha 1$ transcription factor of Saccharomyces cerevisiae [7]. All other strains included here, however, differed from strain FGSC\# 2489 in that amino acids at positions 32 and 33 were found to be lacking. With the exception of this difference, and a premature stop codon found in the heterothallic taxon phylogenetic species 1 (PS1) resulting in a truncated protein lacking the last three amino acids, the length of the ORF of mat A-1 was identical among all heterothallic and homothallic strains investigated here.

\section{Sequence analysis of mat A-2}

The mat A-2 ORF was successfully amplified and sequenced from all investigated heterothallic mat $A$ strains and the homothallic strains. The nucleotide and amino acid alignments of the gene are shown in Supplemental Figure S1 (Additional file 1), and confirmed the presence of a DNA-binding, acidic amphipathic $\alpha$-helix (HPGdomain) [11]. A number of differences were found when comparing the sequence of genomic DNA and cDNA from strain FGSC \#2489 with the originally published sequence of the same strain [11], and these were also found in all the other strains analyzed, indicating sequencing errors in the original submission.

$N$. intermedia (both subgroups $\mathrm{NiA}$ and $\mathrm{NiB}$ ) and $\mathrm{N}$. crassa (NcC) were found to lack the start codon of mat A-2. No alternative start-codon was found when investigating 40 bp of the upstream sequence, but another methionine was found at aa-position 6 in both these taxa, which may serve as an alternative start-codon. This pattern was confirmed for additional strains of $N$. intermedia (NiA: FGSC\# 8844) and N. crassa (NcC: FGSC\#8865) (data not shown). With these exceptions, the size of the mat A-2 ORF is identical among the heterothallic taxa. However, among the homothallic taxa, the size of the mat A-2 ORF is not conserved. Deletions of up to 6 amino acids are found in $G$. cerealis, G. calospora and N. sublineolata, and, more importantly, in N. dodgei, N. africana, and N. galapagosensis the mat A-2 ORF is disrupted by a stop codon (Figure 1 ). In $N$. dodgei, the stop occurred at codon 39, while in N. africana and N. galapagosensis, the stop was found at codon 288. By sequencing mat A-2 from cDNA of N. dodgei and N. galapagosensis we confirmed the structure of the ORF in these strains. However, in spite of several independent trials, we failed to amplify mat A-2 from cDNA of $N$. africana (data not shown).

\section{Sequence analysis of mat A-3}

We successfully amplified and sequenced mat A-3 from all investigated mat $A$ and homothallic strains, except $N$. terricola, in which this gene previously has been indicated to be absent [15]. The nucleotide and amino acid alignment of mat A-3 is shown in Supplemental Figure S1 (Additional file 1), and shows the presence of an HMG-box. When comparing our DNA sequence obtained from genomic and CDNA of strain FGSC\# 2489 of N. crassa with the original published mat $A-3$ sequence of this strain [11], we found only one silent nucleotide difference. This difference was found in all other strains analyzed here, supporting our revised description of mat A-3.

The length of the ORF was found to be intact in all taxa studied here, except for the homothallic taxa N. africana, N. galapagosensis, N. dodgei and N. lineolata. Sequencing of both genomic and cDNA from these four taxa revealed 
that amino acids 100-177 have been deleted in N. africana and N. galapagosensis, a region spanning the first half of the HMG-box. In N. dodgei, no start codon was found, although a methionine was found after 30 amino acids, which might serve as a start codon for mat A-3 in this taxon. Finally, $N$. lineolata has a stop-codon at position 215 (Supplemental Figure S1, Additional file 1).

\section{Evolutionary pattern among sites}

Figure 2 shows the synonymous substitution rate $\left(d_{S}\right)$ for mat- and non-reproductive genes of the heterothallic and homothallic datasets, obtained through pairwise comparisons between taxa in all possible combinations. We found that $\mathrm{d}_{\mathrm{S}}$ did not differ significantly between mat- and non-reproductive genes within each reproductive class. Furthermore, we found that the synonymous substitution rate of the mat-genes did not differ between heterothallic and homothallic taxa, while for the non-reproductive genes we found a significantly higher $\mathrm{d}_{\mathrm{S}}$ in the homothallic as compared to the heterothallic dataset $(P=0.05$, Mann-Whitney $U$-test).

Six models of variable ratios of nonsynonymous $\left(\mathrm{d}_{\mathrm{N}}\right)$ to synonymous substitution rates $\left(\mathrm{d}_{\mathrm{S}}\right), \omega$, among gene codons (site models outlined by Nielsen and Yang [18] and implemented in PAML version 3.14 [19]) were used to investigate the evolutionary constraints acting on each gene. Detailed descriptions of the models are given in the Methods section. Supplemental Table S1 (Additional file

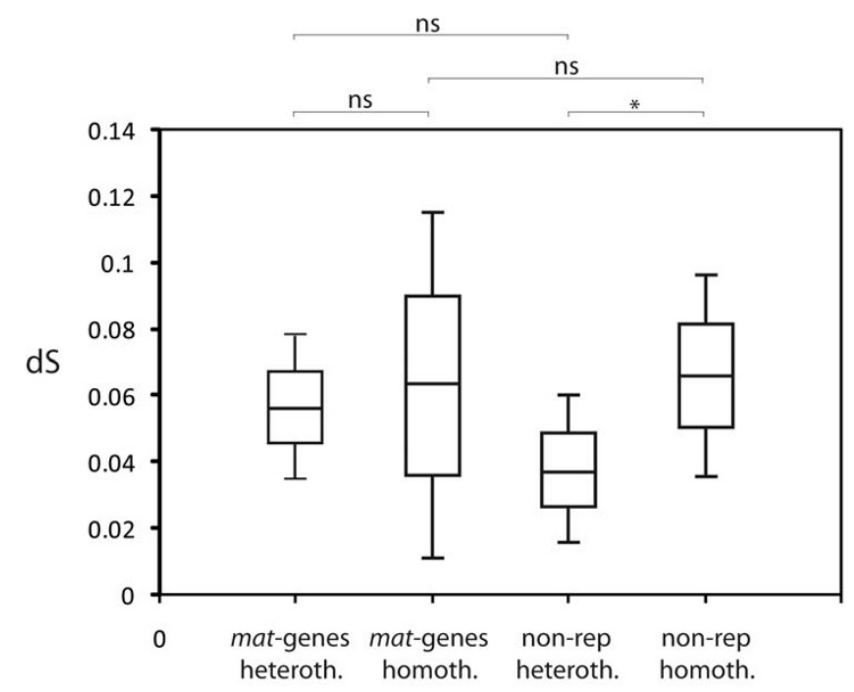

\section{Figure 2}

Mean rate of synonymous substitutions $\left(d_{s}\right)$ for mat- and non-reproductive genes of the heterothallic and homothallic datasets, obtained through pairwise comparisons between taxa in all possible combinations. Heterothallic and homothallic non-reproductive genes are statistically different from each other $(P=0.05$, Mann-Whitney U-test).
1) lists likelihood ratio statistics and parameter estimates for the four mat-genes, and three genes which function is assumed to be independent of reproductive mode of a fungus; act, tef-1 and $c c g-7$ [20-22]. The Bayes Empirical Bayes (BEB) probabilities for each codon of the mat-genes to fit into one of the three classes of sites obtained by running the positive-selection model are shown in Figure 3.

We found a higher $\omega$ among mat-genes than among nonreproductive genes $(P<0.05$, Fisher exact test). Given an equal synonymous substitution rate between mat and non-reproductive genes within each reproductive class (Figure 2), this finding indicates that mat-genes evolve faster than the non-reproductive genes. Furthermore, all four mat-genes were found to evolve faster among the homothallic than the heterothallic taxa; although the synonymous substitution rate of the mat-genes was equal among these groups, the averaged value of $\omega$ for each matgene, obtained using the one-ratio model implemented in PAML version 3.14, was higher in the homothallic compared to the heterothallic dataset $(0.385$ versus 0.144 for mat $a-1,0.487$ versus 0.233 for mat $A-1,0.581$ versus 0.386 for $m a t A-2$ and 0.427 versus 0.316 for $m a t A-3 ; P<$ 0.001 , Fisher exact tests). This pattern was also found for the tef- 1 gene, for which we found a $\omega$ of 0.133 in the homothallic dataset as compared to 0.017 for the heterothallics. In contrast, the $\omega$ between homothallic and heterothallic dataset did not differ for the act or the $c c g-7$ genes. For the act-gene we estimated $\omega$ of 0 for both datasets and for $c c g-7$ we found $\omega$ of 0.102 versus 0.097 for the heterothallic and homothallic datasets, respectively.

Mat $a-1$ was found to be the most conserved mat-gene in both datasets, with lowest average $\omega$ ratios. Although $\omega$ was smaller in the heterothallic than the homothallic dataset, mat $a-1$ was found to evolve under positive selection among the heterothallic taxa (i.e. both models allowing for sites with $\omega>1$ fitted the data significantly better than the corresponding neutral model, $P<0.05$ ). Bayes empirical Bayes (BEB) calculations for posterior probabilities of sites belonging to site classes revealed two codons, codon 7 and 43, with a posterior probability $>0.95$ to belong to the class with $\omega>1$, using both the positiveselection and the beta\& $\omega$ model (Figure 3a).

The sites of mat a-1 showing an elevated $\omega$ were not identical between the heterothallic and homothallic datasets (Figure 3a, b). The second exon was found to be extremely conserved among the heterothallic taxa, and codes for a DNA binding HMG-domain. The PRPPNAYILYRK motif suggested by Staben \& Yanofsky [8] to be functionally important for mating, based on conservation between Schizosaccharomyces pombe and N. crassa, was found to be completely conserved in both heterothallic and homothallic taxa possessing this gene, but variation was found 
a) mat $a-1$, heterothallic taxa, classes of w: $\square 0.03 \quad \square 1.00 \quad \square 5.20$

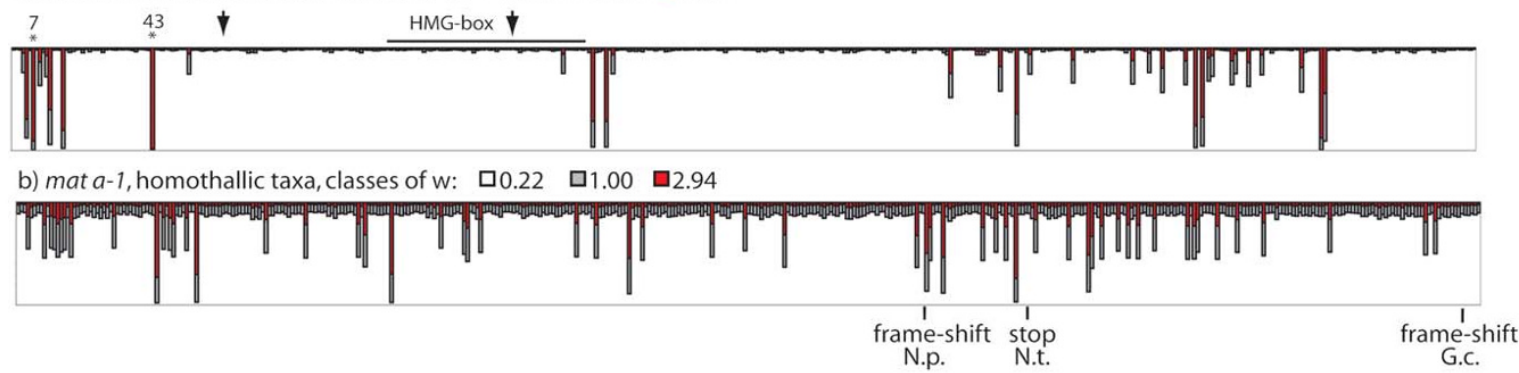

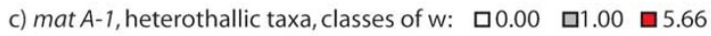

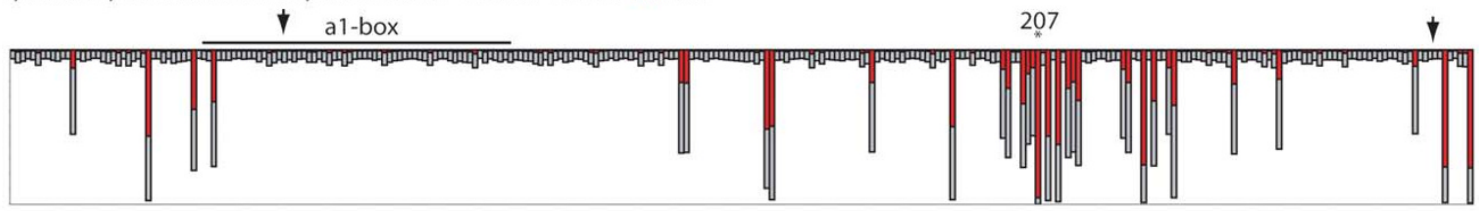

d) mat A-1, homothallic taxa, classes of w: $\quad$ a0.00 미 $\quad$ ⒈00
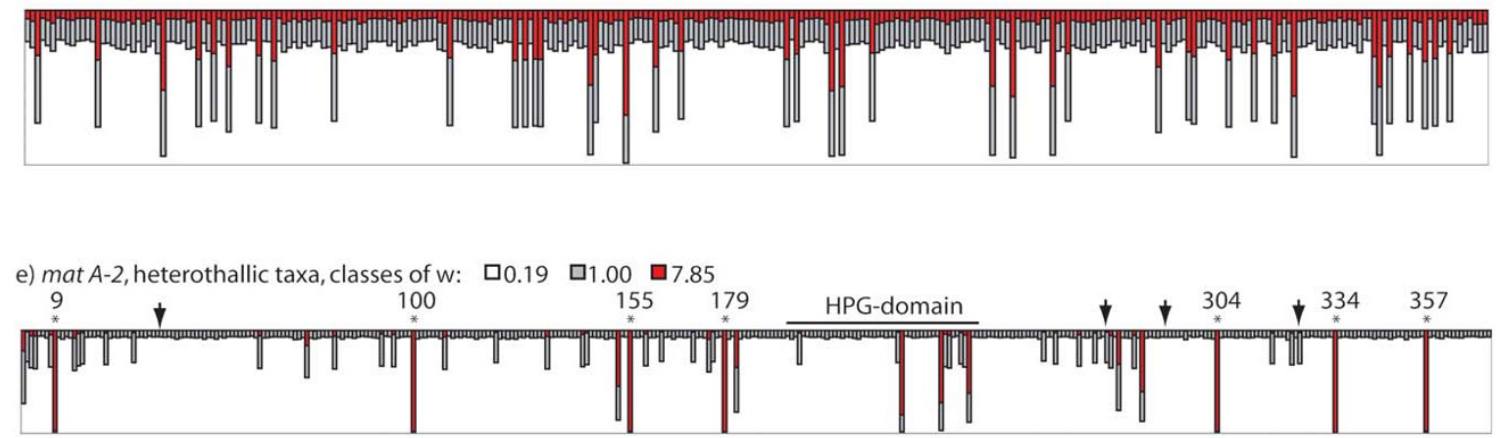

f) mat A-2, homothallic taxa, classes of w: $\quad$ 0.00 ㅁ1.00 ㅁ1.04
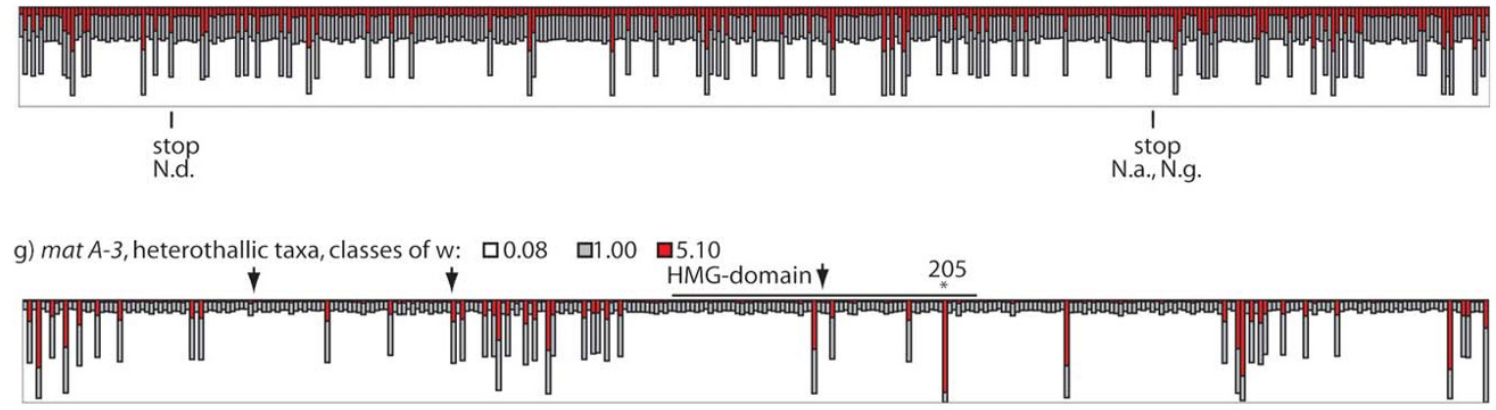

h) mat A-3, homothallic taxa, classes of $\mathrm{w}$ : $\quad$ 0.17 ㅁ1.00 $\quad$ ⒉11

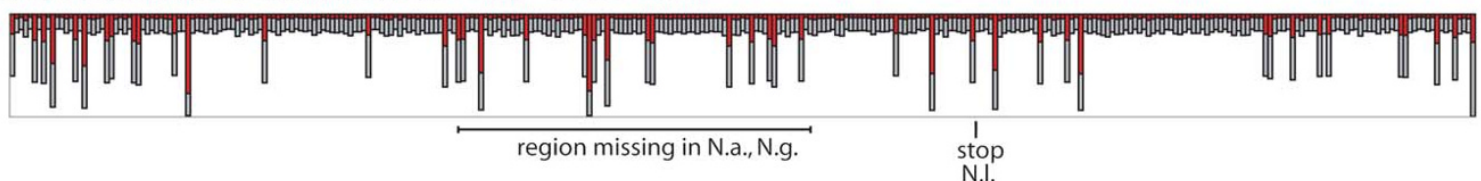

\section{Figure 3}

Probabilities that sites of the mat-genes belong to site classes with different selective pressures. Bar height represents the BEB probabilities for each site to belong to one of the three site classes obtained by running model $2 \mathrm{a}$ in PAML version 3.14. Asterisks indicate a probability of $>95 \%$ that the site belongs to a class with $\omega>I$ in the positive-selection and/or B\& $\omega$-models implemented in codeml-package, with site number as in amino acid alignment in Supplemental Figure SI. Arrow indicate intron splicing positions and pins indicate disrupted ORFs in N.a. (N. africana), N.g. (N. galapagosensis), N.t. (N. terricola), N.I. (N. lineolata), G.c. (G. calospora), N. p. (N. pannonica). Functional domains of each gene, as defined by Debuchy and Turgeon [I4], are indicated. 
within the other part of the HMG-box for both groups of taxa (Figure 3a, b).

For mat A-1, the neutral models of evolution fitted the data equally well as the models of positive selection. The rapidly evolving sites in the heterothallic dataset were clustered into domains, e.g. in the C-terminal end of mat $A-1$ we found a stretch of adjacent rapidly evolving sites, including one site with a BEB posterior probability of $P>$ 0.95 to belong to a class with $\omega>1$, while the $\alpha 1$-box was found to be conserved (Figure 3c). This was in contrast to the homothallic dataset in which the rapidly evolving sites are more evenly distributed along the gene (Figure 3d).

In mat $A-2$ we found the most pronounced difference between the heterothallic and homothallic dataset. In the heterothallic dataset, mat A-2 was found to evolve under positive selection. BEB calculations of posterior probabilities revealed seven sites with $P>0.95$ to belong to the class with $\omega>1$ using the positive-selection and the beta\& $\omega$ model (Figure $3 e$ ). This was very different from the homothallics in which a large proportion of sites were found to evolve neutrally; more than half of the codons were found in a class with $\omega=1.011$ using the discrete model. Noteworthy, the discrete model fitted the data significantly better than the one-ratio model for all datasets except the mat A-2 of the homothallics, for which the oneratio model with a $\omega$ of all codons of 0.581 fitted equally well. The HPG-domain that was previously suggested to be conserved among euascomycetes [14] was found to be conserved in our heterothallic dataset, except for three rapidly evolving sites. However, the HPG-domain is not conserved in the homothallic dataset (Figure 3e, f).

In mat A-3, the smallest difference in average $\omega$ between the heterothallics and the homothallics was observed. Similarly to mat $a-1$, and in contrast to the observations at mat A-1 and mat A-2, when using the discrete model no codons were found in a class with $\omega \approx 1$ in the homothallic dataset for mat A-3. The HMG domain was more conserved in the heterothallic than in the homothallic taxa. However, the one site found to be in a class with $P>0.95$ of positive selection (site 205) in the heterothallics was located within this domain.

The investigated region of the act-gene was found to be extremely conserved in both datasets. This was also the case for all codons of tef- 1 for the heterothallic dataset, while a small proportion (3.3\%) of the sites of this gene was found to evolve under positive selection among homothallic taxa (i.e. found in a class with $\omega$ of 6.062 using both the discrete and positive selection model: Supplemental Table S1 (Additional file 1)). For both of these genes, no neutrally evolving codons were identified in any of the datasets. For the $c c g-7$ gene the majority (>90\%) of the sequenced codons are conserved in both datasets, however, a small proportion of the codons was found in a class with $\omega$ of 1.6 in the heterothallic dataset and 1.175 in the homothallic dataset, under the discrete model (Supplemental Table S1, Additional file 1).

\section{Discussion}

In this study we confirm that the mat-genes evolve rapidly in Neurospora, and show that the rapid divergence is due to either adaptive evolution or lack of selective constraint, depending on the reproductive mode of taxa.

A lower overall value of $\omega$ was found for the mat-genes among heterothallic as compared to homothallic taxa. Nevertheless, the two mating type genes mat $a-1$ and mat A-2 were found to evolve under positive selection in heterothallic taxa. Although a high between-taxa nucleotide divergence of reproductive genes in fungi has been observed previously [2-4], this is the first evidence of positive selection of reproductive genes in this kingdom. The finding fits directly with patterns observed for animal reproductive genes, in which essentially all identified genes are characterized by high levels of nonsynonymous divergence and positive selection $[1,23,24]$. None of the positively selected sites were found within the regions encoding known functional domains of the genes, and the biological significance of alterations at the positively selected sites is unknown. MAT a- 1 and its orthologs in other filamentous ascomycetes are characterized by a conserved region similar to motifs found in several DNA binding proteins of the high mobility group (HMG), and has been shown to bind to DNA in in vitro assays [25]. Mat $a-1$ is one of the master genes regulating fertilization in mat a strains of Neurospora, as well as in other species [14]. During the initial phase of mating in heterothallic Neurospora, the trichogynes (female receptive hyphae) grow toward and fuse with male cells of the opposite mating type, and the directed growth is mediated by chemotropic communication between mat-regulated pheromones diffused from male cells and their corresponding receptors on the trichogynes. Competition between individuals could potentially drive adaptation of mat-genes, as indirectly involved in localization of, binding to and fusion between trichogyne and conidia of opposite mating types. An alternative hypothesis would be that the signature of positive selection observed in heterothallic mat $a-1$ and mat A-2 could be related to mating type associated vegetative incompatibility, rather than sexual development. Vegetative incompatibility genes are highly variable, and some have been shown to evolve in a diversifying manner [26].

Our result of mat a-1 in heterothallic taxa confirms previous reports suggesting that an intact HMG domain and 
the presence of a C-terminal tail, although non-specific, are required for mating $[25,27]$; at least the first part of the HMG-domain is under strong purifying selection among heterothallic taxa, while lower levels of conservation are found in the C-terminal tail (Figure 3a). The finding that a group of closely related homothallic taxa lack mat $a-1$ indicate that this gene is not essential for completion of the homothallic sexual cycle, and it is possible that this function of mat $a-1$ is redundant with another gene in the genomes of this group of taxa. The premature stop codons in mat a-1 in the homothallic N. pannonica and N. terricola indicate that this gene may not be required for completing the sexual cycle in a wider range of homothallic taxa. However, in these two taxa the stop codons are found distal to the HMG box, and functional assays have to be performed to verify this hypothesis.

The MAT A-1 protein is characterized by the presence of an $\alpha 1$ domain showing similarity to the $\alpha 1$ transcription factor of Saccharomyces cerevisiae [7]. Mat A-1 is present in all homothallic taxa investigated here, and the only gene in which the ORF is intact in all homothallic taxa, suggesting the possibility that only the $\alpha 1$ transcription factor is essential to promote sexual development. This scenario would mimic the situation in Cryptococcus neoformans, where recent findings show sexual reproduction between isolates with the same mating type [28]. The allele from $N$. africana has been shown previously to function as a mating activator and to confer mating type-associated vegetative incompatibility in $N$. crassa [29] indicating conservation of both these functions in mat A-1 also in homothallic Neurospora. Nevertheless, we found a large proportion of evenly distributed neutrally evolving sites in the homothallic taxa, even in the $\alpha 1$-box that is conserved among the heterothallic taxa, indicating lower selective constraints on this gene in homothallic taxa.

Mat A-2 encodes a protein containing an acidic amphipathic $\alpha$-helix and is unique to filamentous ascomycetes. It is suggested to not be involved in fertilization, but to function in concert with the other MAT A polypeptides to regulate the expression of genes necessary for postfertilization events $[11,12]$, and the positive selection at this gene among heterothallic taxa could possibly be coupled to its role in recognition of opposite mating-type nuclei during sexual development.

The MAT A-3 (and MAT a-1) HMG-domain is a DNAbinding motif found in non-histone chromosomal proteins and transcription factors. In Neurospora, examples of homothallic taxa lacking either mat A-3 or mat $a-1$ are found, and although the structure of the HMG-domain differ between the genes [14] it has been proposed that the loss of mat $a-1$ or mat $A-3$ is compensated by the remaining $\mathrm{HMG}$ encoding gene [30]. N. terricola, which is the only homothallic taxa included here that lacks mat A3 , has a stop codon disrupting the ORF in mat a-1, suggesting that this protein does not function correctly in this taxon, and we have no indications of directional selection in the $N$. terricola mat $a-1$ gene to change function. Furthermore, two of the four taxa lacking the mat $a-1$ gene, $N$. africana and N. galapagosensis, also lack the first half of the MAT A-3 HMG-domain. Thus, we are not able to provide any support for the hypothesis that the essential functions of the lost mat A-3 or mat a-1 are compensated by the remaining HMG encoding gene in Neurospora.

In addition to the mat-genes, tef- 1 also shows a higher $\omega$ among homothallic taxa as compared to heterothallic, and this result implies that the evolution of this gene is also dependent of reproductive mode of the fungi. Interestingly, this elevated $\omega$ is due to a small proportion of positively selected sites among the homothallic taxa. At this point, we have no explanation for the observation, but it implies that tef-1 is involved in sexual development in Neurospora, or that it is affected by genetic linkage through a close physical proximity to the mat locus [31].

In contrast to the heterothallic taxa, rapid evolution of mat-genes in homothallic Neurospora is caused by genetic decay. Instead of being conserved, the mat-genes in homothallic Neurospora evolve under relaxed selective constraints. This is evident from a higher proportion of neutrally evolving codons among the homothallics as well as observations of a disrupted ORF in three of the four mat-genes in homothallic taxa. Comparisons with non-reproductive genes argue that this disparity is matspecific, and not a nonspecific, genome-wide, phenomenon. The observations here provide a parallel to the observations of ecotypes with additional loss-of-function mutations at one or more SI-modifier genes after the evolutionary switch to autogamy in the Arabidopsis thaliana lineage [32], but is the first time low selective constraints of reproductive proteins associated with the switch to homothallism has been reported from fungi.

Our results indicate that we observe an early stage of degeneration of mat-genes in homothallic Neurospora. With the exception of mat A-2 in N. africana, we found that all genes are expressed in homothallic taxa despite frame shifts and stop codons, and the DNA sequences downstream of the disruptions are relatively conserved. This short degeneration time of the mat-genes in homothallic taxa might explain the results where the alterations in the $N$. africana mat A-1 are not sufficient to abolish its functions when assayed in a heterothallic relative [29]. Furthermore, the observation that they have not yet become pseudogenes, in the sense that they do not exhibit degenerative features that prevent their expression [33], indicates a low cost associated with their expression. 
The results presented here indicate that once a Neurospora switches reproductive mode from heterothallic to homothallic, it expresses low selective pressure for maintenance of functional mat-genes. This finding, together with the observation that both heterothallic counterparts of the mat-genes are not required for sexual development in certain taxa $[15,17]$, implies that within homothallic Neurospora, activation of sexual reproduction can be independent of mat a/A interaction. In other filamentous ascomycetes conversion from heterothallic to homothallic mode evidently resides within the mat locus [5] and both idiomorphic counterparts of heterothallic mat-genes are required for homothallic sexual development. In these taxa, the function of mat-genes has been conserved [34]. This study indicates that the transition to homothallism in Neurospora may represent a different paradigm. The exact nature of this difference is not known, but it would involve differences downstream of mat a/A interaction.

If functional mating-type genes are essential for outcrossing in the homothallics, the observed genetic decay may lead to a progressive loss of outcrossing functions and ultimately, to asexuality. However, alternative ways of outcrossing, independent of mat-genes may be possible, as expression of pheromones and receptors can be detected in some strains deleted for the mat locus [35]. Furthermore, it is possible for strains to bypass the need for fertilization by the formation of heterokaryons, which may lead to outcrossing, as done routinely in the laboratory with the homothallic Aspergillus nidulans [36].

\section{Conclusion}

In this study we show that the mat-genes evolve rapidly in Neurospora, and that the rapid divergence is due to either adaptive evolution or lack of selective constraint, depending on the reproductive mode of taxa. In heterothallic Neurospora, the mat-genes evolve in an adaptive manner while once a Neurospora switches reproductive mode from heterothallic to homothallic there is a low selective advantage for maintenance of functional mat-genes. To our knowledge, this is the first example showing that the evolutionary trajectory of reproductive proteins changes after a switch in reproductive behaviour of an organism.

\section{Methods \\ Fungal material}

Thirty-four strains belonging to 21 taxa of Neurospora and Gelasinospora were used in this study (Table 1). Recent studies show that these two genera are closely related and not reciprocally monophyletic [37-39]. Therefore we include homothallic taxa of both genera in this study, and for simplicity refer to all of them as Neurospora. The strains were obtained from the Fungal Genetics Stock Center (University of Kansas Medical Center, Kansas City, KS).

\section{Nucleic acid extraction and complementary DNA (cDNA) construction}

Strains were grown in minimal medium broth [40] with $1 \%$ sucrose for 3 days at $37^{\circ} \mathrm{C}$, after which nucleic acids were extracted as described previously [41]. Total RNA was treated with DNase I according to the manufacturer's protocol (Fermentas, Burlington, Canada). cDNA was synthesized from total RNA by reverse transcription using an oligo(dT) primer $\left(5^{\prime}-\mathrm{T}_{25} \mathrm{VN}-3^{\prime}\right)$ and Moloney Murine Leukemia Virus (M-MLV) reverse transcriptase (Invitrogen), according to the manufacturers instructions.

\section{Selection of loci}

The entire coding regions of the four mat-genes, and partial coding regions of three additional genes; encoding actin (act: 767 bp) [22], translational elongation factor EF-1 alpha (tef-1: 834 bp) [20] and glyceraldehyde 3phosphate dehydrogenase (ccg-7: 753 bp) [21], were investigated in this study. Act, tef-1 and ccg-7 were included for comparative purposes, and selected to represent genes which function is independent of reproductive mode of a fungus. As both physical proximity and expression levels can have an effect on evolutionary rate [42,43], these genes were selected to be located on different chromosomes, and expression levels of tef- 1 and $c c g-7$ have been shown to vary between different treatments $[20,21]$.

\section{PCR amplification}

All included loci were amplified from one selected strain per taxa. Published sequences for mat a, mat $A$, act, tef-1 and $c c g-7$ of $N$. crassa were used as templates for each gene when designing primers. For isolates where amplification of mat A-3 was not possible using primers based on the $N$. crassa gene sequence, a primer walking sequencing strategy was used for searching the $N$. crassa genome sequence [31] for conserved region upstream from mat A-3. All primer sequences and GenBank accession numbers of reference sequences are given as Supplemental Table S2 (Additional file 1). PCR was performed with 1.55 U ThermoWhite Taq DNA polymerase (Saveen \& Werner, Limhamn, Sweden) on a GeneAmp PCR System 9700 (Applied Biosystems, Foster City, CA) according to the manufacturer's instructions.

\section{DNA sequencing}

The PCR products were purified using a PCR purification kit (QIAquick, Qiagen) and sequencing was performed by Macrogen Inc., Seoul, Korea, utilizing ABI 3730 XL automated sequencers (Applied Biosystems, Foster City, CA). Raw sequence data was analyzed using the Sequencher 4.1.4 software (Gene Codes Corporation, Ann Arbor, MI) and BioEdit version 7.0.5.2 [44]. 
Table I: Fungal strains used in this study

\begin{tabular}{|c|c|c|c|c|}
\hline Taxa & FGSC number & Mating system & Mating type & mat-gene analyzed ${ }^{4}$ \\
\hline \multicolumn{5}{|l|}{ Heterothallic taxa: } \\
\hline N. crassa Shear \& Dodge & 2489 & heteroth. & A & - \\
\hline N. crassa Shear \& Dodge & 4200 & heteroth. & $\mathrm{a}$ & - \\
\hline N. crassa Shear \& Dodge (NcA') & 8900 & heteroth. & $A$ & $\mathrm{Al}, \mathrm{A} 2, \mathrm{~A} 3$ \\
\hline N. crassa Shear \& Dodge ( $\left.\mathrm{NcA}^{\prime}\right)$ & 8848 & heteroth. & $\mathrm{a}$ & al \\
\hline N. crassa Shear \& Dodge (NcB') & 8830 & heteroth. & $A$ & $\mathrm{Al}, \mathrm{A} 2, \mathrm{~A} 3$ \\
\hline N. crassa Shear \& Dodge ( $\left.\mathrm{NcB}^{\prime}\right)$ & 8772 & heteroth. & $\mathrm{a}$ & al \\
\hline N. crassa Shear \& Dodge $\left(\mathrm{NcC}^{\prime}\right)$ & 8858 & heteroth. & $A$ & $A 1, A 2, A 3$ \\
\hline N. crassa Shear \& Dodge ( $\left.\mathrm{NcC}^{\prime}\right)$ & 8860 & heteroth. & $\mathrm{a}$ & al \\
\hline $\mathrm{PS}^{2}$ & 8838 & heteroth. & $A$ & $A 1, A 2, A 3$ \\
\hline $\mathrm{PS}^{2}$ & 8835 & heteroth. & $\mathrm{a}$ & al \\
\hline N. sitophila Shear \& Dodge & 8770 & heteroth. & $A$ & $A 1, A 2, A 3$ \\
\hline N. sitophila Shear \& Dodge & 412 & heteroth. & $\mathrm{a}$ & al \\
\hline N. tetrasperma Shear \& Dodge & 8774 & pseudohomoth. ${ }^{3}$ & $A$ & $\mathrm{Al}, \mathrm{A} 2, \mathrm{~A} 3$ \\
\hline N. tetrasperma Shear \& Dodge & 8775 & pseudohomoth. ${ }^{3}$ & $\mathrm{a}$ & al \\
\hline $\mathrm{PSI} \mathrm{I}^{2}$ & 8817 & heteroth. & $A$ & $\mathrm{Al}, \mathrm{A} 2, \mathrm{~A} 3$ \\
\hline$P S I^{2}$ & 8815 & heteroth. & $\mathrm{a}$ & al \\
\hline N. intermedia Tai $\left(\mathrm{NiA}^{\prime}\right)$ & 8901 & heteroth. & $A$ & $\mathrm{Al}, \mathrm{A} 2, \mathrm{~A} 3$ \\
\hline N. intermedia Tai ( $\left.\mathrm{NiA}^{\prime}\right)$ & 8841 & heteroth. & $\mathrm{a}$ & al \\
\hline N. intermedia Tai (NiB') & 8844 & heteroth. & A & $\mathrm{Al}, \mathrm{A} 2, \mathrm{~A} 3$ \\
\hline N. intermedia Tai (NiB') & 8768 & heteroth. & $\mathrm{a}$ & al \\
\hline $\mathrm{PS}^{2}$ & 8847 & heteroth. & $A$ & $\mathrm{Al}, \mathrm{A} 2, \mathrm{~A} 3$ \\
\hline $\mathrm{PS}^{2}$ & 8853 & heteroth. & $\mathrm{a}$ & al \\
\hline N. discreta Perkins \& Raju & 8780 & heteroth. & $A$ & $\mathrm{Al}, \mathrm{A} 2, \mathrm{~A} 3$ \\
\hline N. discreta Perkins \& Raju & 8827 & heteroth. & $\mathrm{a}$ & al \\
\hline \multicolumn{5}{|l|}{ Homothallic taxa containing all mat-genes: } \\
\hline G. calospora (Mouton) Moreau \& Moreau & 958 & homoth. & - & al, $A 1, A 2, A 3$ \\
\hline G. cerealis Dowding & 959 & homoth. & - & $\mathrm{al}, \mathrm{Al}, \mathrm{A} 2, \mathrm{~A} 3$ \\
\hline N. pannonica Krug \& Khan & 7221 & homoth. & - & al, $A 1, A 2, A 3$ \\
\hline $\begin{array}{l}\text { N. sublineolata (Furuya \& Udagawa) von Arx } \\
\text { Homothallic taxon lacking mat A-3: }\end{array}$ & 5508 & homoth. & - & $\mathrm{al}, \mathrm{Al}, \mathrm{A} 2, \mathrm{~A} 3$ \\
\hline N. terricola Gochenaur \& Backus & 1889 & homoth. & - & $\mathrm{al}, \mathrm{Al}, \mathrm{A} 2$ \\
\hline \multicolumn{5}{|l|}{ Homothallic taxa lacking mat $a-l$ : } \\
\hline N. africana Huang \& Backus & 1740 & homoth. & - & $\mathrm{Al}, \mathrm{A} 2, \mathrm{~A} 3$ \\
\hline N. galapagosensis Mahoney \& Backus & 1739 & homoth. & - & $\mathrm{A} 1, \mathrm{~A} 2, \mathrm{~A} 3$ \\
\hline N. dodgei Nelson \& Novak & 1692 & homoth. & - & $\mathrm{Al}, \mathrm{A} 2, \mathrm{~A} 3$ \\
\hline N. lineolata Frederick \& Uecker & 1910 & homoth. & - & $\mathrm{Al}, \mathrm{A} 2, \mathrm{~A} 3$ \\
\hline
\end{tabular}

Intraspecific subgroups and ${ }^{2}$ phylogenetic species designated by Dettman et al. 2003 [47]

${ }^{3}$ Analyzed as heterothallic since it has been shown to occasionally outcross in nature [49].

${ }^{4} \mathrm{ORF}$ included in evolutionary analyses.

\section{Codon-based likelihood analyses}

Likelihood-based tests were used to investigate the nature of evolutionary pressure acting on the mat-genes in $\mathrm{Neu}$ rospora, using the CODEML program of the PAML version 3.14 package $[19,45]$. All analyses were carried out using separate datasets consisting of the heterothallic or homothallic taxa, as well as a dataset containing both hetero- and homothallics. For each model, equilibrium codon frequencies were estimated from the average nucleotide frequencies at each codon position, amino acid distances were assumed to be equal, and the transition/ transversion ratio $(\kappa)$ was estimated from the data. For all other parameters, we use the default settings provided by Yang et al. [46]. We assumed linkage between collinear sites. The tree structure file used as input file in PAML was:
(((G_calospora,

G_tetrasperma),((N_africana, N_galapagosensis), N_dodgei, N_lineolata), G_cerealis, N_terricola, N_pannonica, $(((()(($ N_crassaA, N_crassaB $)$, N_crassaC $)$, PS3), $\quad$ N_sitophila), $\quad$ N_tetrasperma), PS1),((N_intermediaA, N_intermedia B), PS2)), N_discreta)); shown in Figure 1 and constructed based on the topology obtained from previous reports where neighbor-joining, maximum parsimony and bayesian methods were used to construct species-phylogenies based on multiple loci $[37-39,47]$. We assume the true evolutionary history of the genes under study is the same as the history of the species. When only a subset of the taxa was used in the analyses, the corresponding subset of the topology was used. Sequences downstream of stop codons and 
frameshift mutations were omitted from the analyses. We verified that including the sequence downstream of the disruption did not affect the result by performing parallel runs, in which stop codons and frameshift mutations were omitted form the sequences.

We estimated the synonymous substitution rate $\left(\mathrm{d}_{\mathrm{S}}\right)$ for each gene by comparing rates between taxa in all possible combinations (runmode $=-2$ in PAML 3.14 [19]). A Mann-Whitney U-test (Statistica 7.1, StatSoft, Tulsa, OK) was utilized to examine whether the synonymous substitution pattern differs between mat- and non-reproductive genes in heterothallic and homothallic taxa.

Models of variable ratios of nonsynonymous $\left(\mathrm{d}_{N}\right)$ to synonymous substitution rates $\left(\mathrm{d}_{\mathrm{S}}\right), \omega$, among sites were used to test for the presence of sites under different evolutionary constraints, and to identify them. We used six models outlined by Nielsen and Yang [18] and implemented in PAML version 3.14 [19]. The one-ratio model (Nssites 0) assumes one $\omega$ for all sites, while the discrete model (Nssites 3) uses a general discrete distribution with three site classes estimated from the data. The nearly-neutral model (Nssites 1a) assumes two classes of sites in the protein, with $\omega<1$ and $\omega=1$. The positive-selection model (Nssites 2a) adds a third class of sites to the neutral model, in which $\omega>1$. The beta model (Nssites 7) uses a $\beta$ distribution of $\omega$ over sites: $\beta(p, q)$, which, depending on the parameters $p$ and $q$, can take various shapes in the interval $(0,1)$. The beta\& $\omega$ model (Nssites 8 ) adds an extra class of sites to the beta model, with a proportion of $\omega$ estimated from the data.

To verify which of the models best fits the data, likelihood ratio tests (LRTs) were performed by comparing twice the difference in $\log$-likelihood values $(-2 \ln \Delta)$ between two models using a $\chi^{2}$ distribution, with the number of degrees of freedom equal to the differences in the number of parameters between the models. For each dataset, we made three comparisons: first, the one-ratio model (Nssites 0) was compared to the discrete model (Nssites 3 ), to test for a variation of $\omega$ among codons within the gene. To test whether a gene evolve under positive selection, the two models including a class of codons with positively selected sites (i.e. $\omega>1$; models Nssites $2 \mathrm{a}$ and Nssites 8) were compared to their corresponding neutral models (Nssites 1a and Nssites 7, respectively) using 4, 2 and 2 degrees of freedom, respectively [45].

We identified particular sites in the genes that were likely to have evolved under positive selection by using the Bayes empirical Bayes (BEB) calculation of posterior probabilities for site classes implemented in the positive-selection- and beta\& $\omega$ models [48].

\section{Authors' contributions}

Lotta Wik carried out the majority of the molecular work, participated in sequence alignment and helped to draft the manuscript. Magnus Karlsson participated in the in the design of the study, the molecular work, the statistical analyses and helped to draft the manuscript. Hanna Johannesson initiated the study, performed the statistical analyses and drafted the manuscript. All authors read and approved the final manuscript.

\section{Additional material}

\section{Additional File 1}

Supplemental Table S1. Likelihood ratio statistics and parameter estimates for the two datasets as inferred under six models of $\omega$ over codons. Supplemental Table S2. Primers used in the current study. Supplemental Figure S1. Nucleotide and amino acid alignments of all genes and strains investigated in this study, and the originally published sequence of the

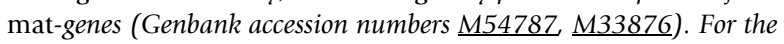
mat-genes, the entire coding sequence is aligned, while for the non-reproductive genes the alignment includes the part of the coding region amplified with primers given in the Supplemental Table S2.

Click here for file

[http://www.biomedcentral.com/content/supplementary/14712148-8-109-S1.pdf]

\section{Acknowledgements}

We wish to thank Timothy Y. James, Henrik Johannesson and Anne Pringle for helpful suggestions and critical reading of the manuscript. Four anonymous reviewers are acknowledged for valuable criticism of the manuscript. Financial support was obtained from the Swedish Research Council, Carl Tryggers Stiftelse för Vetenskaplig Forskning and the Sven and Lily Lawski Foundation.

\section{References}

I. Clark NL, Aagaard JE, Swanson WJ: Evolution of reproductive proteins from animals and plants. Reproduction 2006, I 3 | (I): | | -22.

2. Pöggeler S: Phylogenetic relationships between mating-type sequences from homothallic and heterothallic ascomycetes. Curr Genet 1999, 36(4):222-23I.

3. Turgeon BG: Application of mating type gene technology to problems in fungal biology. Annu Rev Phytopathol I998, 36:115-137.

4. Pöggeler $S$, Kuck $U$ : Identification of transcriptionally expressed pheromone receptor genes in filamentous ascomycetes. Gene 200I, 280(I-2):9-17.

5. Yun SH, Berbee ML, Yoder OC, Turgeon BG: Evolution of the fungal self-fertile reproductive life style from self-sterile ancestors. Proc Natl Acad Sci USA 1999, 96( I 0):5592-5597.

6. Metzenberg RL, Glass NL: Mating type and mating strategies in Neurospora. Bioessays 1990, I2(2):53-59.

7. Glass NL, Grotelueschen J, Metzenberg RL: Neurospora crassa a mating-type region. Proc Natl Acad Sci USA 1990, 87( 13):4912-4916.

8. Staben C, Yanofsky C: Neurospora crassa A mating-type region. Proc Natl Acad Sci USA 1990, 87(13):4917-4921.

9. Picard M, Debuchy R, Coppin E: Cloning the mating types of the heterothallic fungus Podospora anserina - developmental features of haploid transformants carrying both mating types. Genetics 199I, I 28(3):539-547.

10. Turgeon BG, Bohlmann H, Ciuffetti LM, Christiansen SK, Yang G, Schafer W, Yoder OC: Cloning and analysis of the mating-type 
genes from Cochliobolus heterostrophus. Mol Gen Genet 1993 , 238(I-2):270-284.

11. Ferreira AVB, Saupe S, Glass NL: Transcriptional analysis of the mtA idiomorph of Neurospora crassa identifies two genes in addition to mt A-I. Mol Gen Genet 1996, 250(6):767-774.

12. Ferreira AVB, An ZQ, Metzenberg RL, Glass NL: Characterization of mat A-2, mat A-3 and Delta matA mating-type mutants of Neurospora crassa. Genetics 1998, 148(3): 1069-1079.

13. Knop M: Evolution of the hemiascomycete yeasts: on life styles and the importance of inbreeding. Bioessays 2006 , 28(7):696-708.

14. Debuchy R, Turgeon BG: Mating-type structure, evolution, and function in Euascomycetes. In The Mycota l: Growth, Differentiation and Sexuality Edited by: Kues, Fischer. Berlin Heidelberg: SpringerVerlag; 2006:293-323.

15. Beatty NP, Smith ML, Glass NL: Molecular characterization of mating-type loci in selected homothallic species of Neurospora, Gelasinospora and Anixiella. Mycol Res 1994 98:1309-1316.

16. Glass NL, Vollmer SJ, Staben C, Grotelueschen J, Metzenberg RL, Yanofsky C: DNAs of the two mating-type alleles of Neurospora crassa are highly dissimilar. Science 1988, 241 (4865):570-573.

17. Glass NL, Metzenberg RL, Raju NB: Homothallic Sordariaceae from nature - the absence of strains containing only the a mating type sequence. Exp Mycol 1990, I 4(3):274-289.

18. Nielsen R, Yang ZH: Likelihood models for detecting positively selected amino acid sites and applications to the HIV-I envelope gene. Genetics 1998, 148(3):929-936.

19. Yang Z: PAML: a program package for phylogenetic analysis by maximum likelihood. Comput Appl Biosci 1997, I3(5):555-556.

20. Ichi-Ishi A, Inoue $\mathrm{H}$ : Cloning, nucleotide sequence, and expression of tef- $I$, the gene encoding translation elongation factor I $\alpha$ (EF-I $\alpha$ ) of Neurospora crassa. Jpn J Genet 1995, 70:273-287.

21. Shinohara ML, Loros JJ, Dunlap J: Glyceraldehyde-3-phosphate dehydrogenase is regulated on a daily basis by the circadian clock. Biol Chem 1998, 273(I):446-452.

22. Tinsley J, Lee I, Minke P, Plamann M: Analysis of actin and actinrelated protein 3 (ARP3) gene expression following induction of hyphal tip formation and apolar growth in $\mathrm{Neu}$ rospora. Mol Gen Genet 1998, 259:601-609.

23. Vacquier VD: Evolution of gamete recognition proteins. SCience | 998, 28 I(5385): 1995- 1998.

24. Swanson WJ, Vacquier VD: The rapid evolution of reproductive proteins. Nat Rev Genet 2002, 3(2): | 37- I 44.

25. Philley ML, Staben C: Functional analyses of the Neurospora crassa Mt a-I mating-type polypeptide. Genetics 1994 137(3):7| 5-722

26. $\mathrm{Wu} \mathrm{Cl}$, Saupe S, Glass L: Evidence for balancing selection operating at the het-c heterokaryon incompatibility locus in a group of filamentous fungi. Proc Natl Acad Sci USA 1998 95(2I): $12398-12403$.

27. Arnaise S, Zickler D, Glass NL: Heterologous expression of mating-type genes in filamentous fungi. Proc Natl Acad Sci USA 1993, 90(I4):6616-6620.

28. Lin X, Hull CM, Heitman J: Sexual reproduction between partners of the same mating type in Cryptococcus neoformans. Nature 2005, 434:1017-1021.

29. Glass NL, Smith ML: Structure and function of a mating-type gene from the homothallic species Neurospora africana. Mol Gen Genet 1994, 244(4):40 I-409.

30. Robertson SJ, Bond DJ, Read ND: Homothallism and heterothallism in Sordaria brevicollis. Mycol Res 1998, 102:1215-1223.

31. Neurospora crassa Database, Broad Institute [http:// www.broad.mit.edu/annotation/genome/neurospora/Home.html].

32. Nasrallah ME, Liu P, Sherman-Broyles S, Boggs NA, Nasrallah JB: Natural variation in expression of self-incompatibility in Arabidopsis thaliana: implications for the evolution of selfing. Proc Natl Acad Sci USA 2004, I 0 I(45): 16070-16074.

33. Balakirev ES, Ayala FJ: Pseudogenes: are they "junk" or functional DNA? Ann Rev Genet 2003, 37:123-151.

34. Lee J, Lee T, Lee YW, Yun SH, Turgeon BG: Shifting fungal reproductive mode by manipulation of mating type genes: obligatory heterothallism of Gibberella zeae. Mol Microbiol 2003, 50(I): | $45-152$.
35. Paoletti M, Seymour FA, Alocert MJC, Kaur N, Calvo AM, Archer DB, Dyer PS: Mating type and the genetic basis of self-fertility in the model fungus Aspergillus nidulans. Curr Biol 2007, 17:1384-1389.

36. Hoffman B, Eckert SE, Krappman S, Braus GH: Sexual diploids of Aspergillus nidulans do not form by random fusion of nuclei in the heterokaryon. Genetics 200I, 157:|4|-147.

37. Dettman JR, Harbinski FM, Taylor JW: Ascospore morphology is a poor predictor of the phylogenetic relationships of Neurospora and Gelasinospora. Fungal Genet Biol 200I, 34(I):49-6I.

38. Garcia D, Stchigel AM, Cano J, Guarro J, Hawksworth DL: A synopsis and re-circumscription of Neurospora (syn. Gelasinospora) based on ultrastructural and 285 rDNA sequence data. Mycol Res 2004, 108: III9-II42.

39. Cai L, Jeewon R, Hyde KD: Phylogenetic investigations of Sordariaceae based on multiple gene sequences and morphology. Mycol Res 2006, I I 0:137-150

40. Vogel H: Distribution of lysine pathways among fungi: evolutionary implications. Am Nat 1964, 98:435-466.

4I. Karlsson M, Olson A, Stenlid J: Expressed sequences from the basidiomycetous tree pathogen Heterobasidion annosum during early infection of Scots pine. Fungal Genet Biol 2003, 39(I):5I-59.

42. Higgs PG, Hao W, Golding GB: Identification of conflicting selective effects on highly expressed genes. Evol Bioinf 2007, 2: I-I3.

43. Sällström B, Arnaout RA, Davids W, Bjelkmar P, Andersson S: Protein evolutionary rates correlate with expression independently of synonymous substitutions in Helicobacter pylori. J Mol Evol 2006, 62:600-6/4.

44. Hall TA: BioEdit: a user-friendly biological sequence alignment editor and analysis program for windows 95/98/NT. Nucl Acid Symp Ser 1999, 41:95-98.

45. Yang ZH, Nielsen R, Goldman N, Pedersen AMK: Codon-substitution models for heterogeneous selection pressure at amino acid sites. Genetics 2000, I55(I):431-449.

46. Yang ZH, Swanson WJ, Vacquier VD: Maximum-likelihood analysis of molecular adaptation in abalone sperm lysin reveals variable selective pressures among lineages and sites. Mol Biol Evol 2000, 17(10): | 446- I 455.

47. Dettman JR, Jacobson DJ, Taylor JW: A multilocus genealogical approach to phylogenetic species recognition in the model eukaryote Neurospora. Evolution 2003, 57( I 2):2703-2720.

48. Yang Z, Wong WS, Nielsen R: Bayes empirical bayes inference of amino acid sites under positive selection. Mol Biol Evol 2005, 22(4): $1107-1118$.

49. Powell AJ, Jacobson DJ, Natvig DO: Allelic diversity at the het-c locus in Neurospora tetrasperma confirms outcrossing in nature and reveals an evolutionary dilemma for pseudohomothallic ascomycetes. J Mol Evol 200I, 52(I):94-102.

Publish with Biomed Central and every scientist can read your work free of charge

"BioMed Central will be the most significant development for disseminating the results of biomedical research in our lifetime. "

Sir Paul Nurse, Cancer Research UK

Your research papers will be:

- available free of charge to the entire biomedical community

- peer reviewed and published immediately upon acceptance

- cited in PubMed and archived on PubMed Central

- yours - you keep the copyright
BioMedcentral 